\title{
Protective Effect of Yi Shen Pai Du Formula against Diabetic Kidney Injury via Inhibition of Oxidative Stress, Inflammation, and Epithelial-to-Mesenchymal Transition in $d b / d b$ Mice
}

\author{
Qilin Zhang, ${ }^{1,2}$ Xiaocui Liu, ${ }^{3}$ Mitchell A. Sullivan, ${ }^{4}$ Chen Shi $\mathbb{D}^{1,2}$ and Bin Deng $\mathbb{D}^{1,2}$ \\ ${ }^{1}$ Department of Pharmacy, Union Hospital, Tongji Medical College, Huazhong University of Science and Technology, \\ 430030 Wuhan, China \\ ${ }^{2}$ Hubei Province Clinical Research Center for Precision Medicine for Critical Illness, 430030 Wuhan, China \\ ${ }^{3}$ Department of Pharmacy, Taihe Hospital, Hubei University of Medicine, No. 32 South Renmin Road, Huibei, \\ Shiyan 442000, China \\ ${ }^{4}$ Glycation and Diabetes Group, Mater Research Institute-the University of Queensland, Translational Research Institute, Brisbane, \\ Queensland 4072, Australia
}

Correspondence should be addressed to Chen Shi; 29136909@qq.com and Bin Deng; dengbin@hust.edu.cn

Received 13 April 2021; Revised 3 August 2021; Accepted 12 August 2021; Published 31 August 2021

Academic Editor: Liang-Jun Yan

Copyright ( 2021 Qilin Zhang et al. This is an open access article distributed under the Creative Commons Attribution License, which permits unrestricted use, distribution, and reproduction in any medium, provided the original work is properly cited.

\begin{abstract}
Objective. Diabetic kidney disease (DKD) is one of the most common chronic microvascular complications of diabetes; however, there remains a lack of effective therapeutic strategies. Yi Shen Pai Du Formula (YSPDF), a traditional Chinese medicine preparation, has been clinically used in treating chronic kidney disease (CKD) for more than 20 years. However, whether YSPDF has a therapeutic effect on DKD has not been studied. Methods. This study was conducted to investigate the effect of YSPDF administration on $d b / d b$ mice, a model of type 2 diabetes that develops DKD, and reveal its underlying mechanism of action through a high glucose- (HG-) induced renal injury cell model. Results. We found that YSPDF significantly improved numerous biochemical parameters (fasting blood glucose, serum creatinine, blood urea nitrogen, $24 \mathrm{~h}$ urine total protein, total cholesterol, and total triglycerides) and ameliorated the abnormal histology and fibrosis of renal tissue. Moreover, the status of oxidative stress and levels of inflammatory cytokines (TNF- $\alpha$, IL-6, IL-1 $\beta$, and MCP-1) were markedly inhibited by YSPDF treatment. YSPDF treatment significantly mitigated renal fibrosis, with evidence suggesting that this was by inhibiting epithelial-to-mesenchymal transition (EMT) via suppression of the TGF- $\beta 1 / \mathrm{Smad}$ pathway. Interestingly, the expression of Nrf2, HO-1, and NQO1, proteins known to be associated with oxidative stress, were significantly increased upon administration of YSPDF. The levels of NLRP3 inflammasome proteins, including NLRP3, ASC, caspase-1, and cleaved caspase-1 were decreased in the YSPDF-treated group. Cell experiments showed that YSPDF inhibited EMT and the NLRP3 inflammasome in HG-exposed HK-2 cells, possibly via activation of Nrf2. Conclusion. Our study indicates that YSPDF may ameliorate renal damage in $d b / d b$ mice via inhibition of oxidative stress, inflammation, and EMT, with the mechanism potentially being related to the activation of the Nrf2 pathway.
\end{abstract}

\section{Introduction}

Diabetic kidney disease (DKD), also known as diabetic nephropathy (DN), is one of the most common chronic microvascular complications of diabetes and is the leading cause of end-stage renal failure (ESRD). The growing prevalence of diabetes has resulted in a rapid increase in the global incidence of $\mathrm{DKD}$, bringing an enormous economic burden to society $[1,2]$. The pathogenesis of DKD has not been fully elucidated, and currently, there are no effective treatments 
[3]. Thus, developing highly effective and low-toxic drugs that can prevent the occurrence and progression of DKD has become a serious medical challenge.

Tubulointerstitial fibrosis is a crucial pathological alteration underlying the progression of $\operatorname{DKD}[4,5]$. Epithelialto-mesenchymal transition (EMT), characterized by a loss of epithelial phenotype and a gain of profibrotic features, plays a crucial role in the development and progression of tubulointerstitial fibrosis [4]. Accumulating evidence indicates that EMT accelerates the generation of renal fibrosis in DKD. When EMT is activated, the kidney fibroblasts have been shown to translate into myofibroblasts, secreting excessive extracellular matrix (ECM) and eventually causing the development of fibrosis [5]. EMT is regulated by many factors. It has been demonstrated that transforming growth factor- $\beta 1$ (TGF- $\beta 1$ ) is able to induce EMT activation in renal tubular epithelial cells [6, 7]. TGF- $\beta 1$ phosphorylates downstream proteins Smad2 and Smad3, increasing the expression level of $\alpha$-smooth muscle actin ( $\alpha$-SMA) and decreasing the expression level of E-cadherin [3]. Therefore, inhibiting EMT via the TGF- $\beta 1 /$ Smad pathway might be an effective therapeutic target for DKD.

It has been widely reported that oxidative stress and an inflammatory response are implicated in DKD $[8,9]$. High glucose not only promotes generation of reactive oxygen species (ROS) by inhibiting the nuclear factor-erythroid 2related factor 2 (Nrf2) pathway but also activates the nucleotide binding and oligomerization domain-like receptor family pyrin domain-containing 3 (NLRP3) [10-12]. Key proteins of the Nrf2 pathway, Nrf2, HO-1, and NQO1, have been shown to be significantly reduced in DKD mice, indicating that the Nrf2 pathway may protect the kidney from damage by decreasing oxidative stress [13]. When the NLRP3 inflammasome is activated, the secretion of proinflammatory cytokines IL-18 and IL- $1 \beta$ is increased, with kidney inflammation and fibrosis being amplified [14]. A knockout of NLRP3 significantly attenuated the inflammatory response of renal tissue and improved renal function in a DKD mouse model [15]. Moreover, it has been reported that the renal NLRP3 inflammasome can also be activated by elevating levels of ROS [16]. In summary, reduction of oxidative stress and inflammation could be effective approaches to attenuate kidney injury induced by hyperglycemia.

Astragali radix (Huang Qi) and Rhei radix et rhizome (Da Huang) is a classical formula for treating kidney injury [17]. YSPDF is an innovative Chinese medicine prescribed on the basis of containing Astragali radix and Rhei radix et rhizome. It also contains two additional traditional Chinese medicines: Hirudo (Shui Zhi) and Bombyx batrytocatus (Jiang Can). The pharmacological effect of YSPDF on chronic kidney disease (CKD) has been systematically studied; however, whether YSPDF has a therapeutic effect on DKD has not been investigated [18]. In the prescription of YSPDF, Astragali radix is a "Jun medicine" with the traditionally described function of replenishing qi. It has been reported that the nephritis prescription composed of Astragalus membranaceus and Angelica sinensis could significantly reduce urinary albumin and serum creatinine hydration in DN rats and protect renal function [19]. Rhei radix is a
"Chen medicine" with the traditionally described effect of cleaning away heat, purgation, and detoxification. Modern pharmacology studies have shown that the extract of Rhei radix could effectively inhibit renal oxidative stress, lipid peroxidation, and ECM accumulation to reduce diabetic kidney damage [20,21]. Hirudo and Bombyx batrytocatus were the adjuvant medicine, whose function were promoting blood circulation to remove blood stasis and eliminating wind. Studies have shown that hirudin in Hirudo has effects as an anticoagulant and antithrombotic, which can inhibit renal fibrosis by reducing the levels of renal inflammatory factors such as IL-1 and IL-6 and renal tubular EMT [22]. Thus, according to the current literature, we propose a hypothesis that YSPDF may have a positive effect on the treatment of $\mathrm{DKD}$, and its mechanism may be related to inhibiting renal EMT, oxidative stress, and inflammation.

In the present study, we systematically investigated the protective effect of YSPDF on diabetic kidney injury in $d b / d b$ mice, aiming to reveal potential mechanisms. This work will provide new knowledge on the pharmacological effects of YSPDF and help determine if YSPDF could be used as a clinical therapy for DKD.

\section{Materials and Methods}

2.1. Preparation of Yi Shen Pai Du Formula (YSPDF). YSPDF was prepared using extractions from four traditional Chinese medicines: Astragali radix (Huang Qi) (lot no. 20170516), Rhei radix et rhizome (Da Huang) (lot no. 20170411), Hirudo (Shui Zhi) (lot no. 20170520), and Bombyx batrytocatus (Jiang Can) (lot no. 20170510) (Table 1). All the botanical and animal names are recorded and can be validated using http://mpns.kew.org/mpns-portal/?_ga= 1.111763972.1427522246.1459077346. Crude YSPDF was purchased from Bozhou Jinshaotang Chinese Medicine Decoction Co., Ltd. The ratio of Astragali radix, Rhei radix et rhizome, Hirudo, and Bombyx batrytocatus in the formula was $25: 5: 3: 3$. This crude YSPDF was powdered and subjected to reflux extraction with 10 volumes of water for $1.5 \mathrm{~h}$. The aqueous extracts were filtered and collected. The extraction was repeated twice with the method described above, and then, all the extracts were evaporated to dryness under reduced pressure. Finally, $1 \mathrm{~g}$ of YSPDF extracts were equivalent to $1.44 \mathrm{~g}$ of original crude material. The pulverized YSPDF extracts were dispersed and dissolved in distilled water for animal experiments.

The quality of the extracted YSPDF was analyzed using reversed phase high-performance liquid chromatography (RP-HPLC) on the basis of a method established by the Department of Pharmacy, Union Hospital, Tongji Medical College, Huazhong University of Science and Technology (Wuhan, China) [23]. The HPLC chromatogram was obtained from the HPLC unit (Agilent 1260, USA) and Agilent TC-C18 column $(4.6 \mathrm{~mm} \times 250 \mathrm{~mm}, 5 \mu \mathrm{m}$ particle size $)$ with the temperature being maintained at $30^{\circ} \mathrm{C}$. The mobile phase consisted of solutions with varying ratios of $\mathrm{A}$ (acetonitrile) and $B(0.2 \%$ formic acid $)$ at a flow rate of $1.0 \mathrm{~mL} / \mathrm{min}$ and the detection wavelength was at $260 \mathrm{~nm}$. A linear gradient elution was performed with the gradient procedure as 
TABLE 1: The components and ratio of YSPDF.

\begin{tabular}{lccc}
\hline Pharmaceutical name & $\begin{array}{c}\text { Botanical plant name/animal medicinal } \\
\text { materials animal name }\end{array}$ & Family & Medicinal part \\
\hline Astragali Radix & $\begin{array}{c}\text { Astragalus membranaceus (Fisch). Bge. } \\
\text { var. mongholicus (Bge). Hsiao }\end{array}$ & Leguminosae & Root \\
Rhei radix et rhizoma & Rheum palmatum L. & Polygonaceae & Root and rhizome \\
Hirudo & Whitmania pigra Whitman & Hirudinidae & Drying body \\
Bombyx batrytocatus & Bombyx mori Linnaeus & Bombycidae & Drying body \\
\hline
\end{tabular}

follows: $0-20 \mathrm{~min}, 20 \%-40 \% \mathrm{~A} ; 20-30 \mathrm{~min}, 40 \% \mathrm{~A} ; 30-$ $35 \mathrm{~min}, 90 \% \mathrm{~A} ; 35-40 \mathrm{~min}, 20 \% \mathrm{~A}$. A standard was created that contained the following: calycosin glucoside, aloe-emodin, rhein, emodin, chrysophanol, and physcion.

2.2. Animals Experiment. Male mice on a C57BL/6JNju background (8 weeks) were purchased from the Hubei Provincial Center for Food and Drug Safety. Male mice on C57BL/BKS-Lepr ${ }^{\mathrm{em} 2 \mathrm{Cd} 479} / \mathrm{Nju}$ (C57BL/6JNju- $d b / d b$, genotyping is (Lepr $\left.{ }^{\mathrm{db}}\right)$ mut/mut) background ( 8 weeks) were purchased from the Model Animal Research Center of Nanjing University. Mice were bred in an SPF room with standard cages ( 4 mice/cage) under the following conditions: $22 \pm 1{ }^{\circ} \mathrm{C}$ and a $12 \mathrm{~h}$ dark-light cycle. All animals had ad libitum access to water and standard chow $(6 \% \mathrm{kcal}$ from fat, 14.3 $\mathrm{MJ} \mathrm{kg}^{-1}$, Hubei Provincial Center for Disease Control and Prevention). All animal experiments were approved (no. 2019S938) by the institutional Animal Care and Use Committee of Tongji Medical College, Huazhong University of Science and Technology. The animal care and experimental procedures were carried out in accordance with the Guidelines of the Institutional Animal Care and Use Committee of Tongji Medical College and the National Institutes of Health Guide for the Care and Use of Laboratory Animals.

After one week acclimatization, 10 healthy C57BL/6 mice and $20 \mathrm{db} / \mathrm{db}$ mice were divided into 3 groups: Group I (NC): healthy mice, Group II (DKD): $d b / d b$ mice, Group III (DKD+YSPDF): $d b / d b$ mice were administered intragastrically with $0.4 \mathrm{~mL}$ of YSPDF $(2 \mathrm{~g} / \mathrm{kg})$ once daily. The usage of YSPDF in this study was determined based on the clinical usage of YSPDF and previous animal study [24]. Mice in the $\mathrm{NC}$ and DKD groups were administered intragastrically with an equal volume of distilled water. Drug treatment lasted for 8 weeks until the sacrifice of the mice.

2.3. Sample Collection and Metabolic Index Detection. Before the mice were sacrificed, they were placed in metabolic cages for 1 day. Urine samples were collected over the 24 hours for the analysis of urinary creatinine (UCr) and $24 \mathrm{~h}$ urine total protein (24h UTP). At the end of the experiment, the mice were fasted overnight and anaesthetized with sodium pentobarbitone ( $150 \mathrm{mg} / \mathrm{kg}$ in traperitoneal). The body weight, liver weight, kidney weight, and fasting blood glucose level (FBG) were measured. The FBG levels were measured by a glucometer $12 \mathrm{~h}$ after fasting at the end of the study. Blood samples of each mouse were collected and immediately separated by centrifugation $\left(1200 \mathrm{~g}, 4^{\circ} \mathrm{C}, 15 \mathrm{~min}\right)$ to obtain the serum. The serum samples were kept for the test of serum creatinine (SCr), blood urea nitrogen (BUN), total cholesterol (TC), total triglycerides (TG), high-density lipoprotein (HDL), and low-density lipoprotein (LDL). The kidney tissues were collected and divided into two parts across the sagittal plane: one part was fixed in $10 \%$ formalin and the rest was stored at $-80^{\circ} \mathrm{C}$.

2.4. Biochemical Analysis. The urinary creatinine (UCr) and SCr were measured by a creatinine assay kit (C011-2-1). The $24 \mathrm{~h}$ urine total protein ( $24 \mathrm{~h}$ UTP) was detected by a urine protein test kit (C035-1-1). The level of BUN (C013-2-1), TG (C110-1-1), TC (C111-1-1), LDL (A113-1-1), and HDL (A112-1-1) in the serum, and the level of ROS (E004-1-1), glutathione (GSH, A006-2-1), malondialdehyde (MDA, A003-1-2), superoxide dismutase (SOD, A001-3-2), and catalase (CAT, A007-1-1) in the kidney tissues were determined by ELISA kits (Nanjing Jian Cheng Bioengineering Institute, China), respectively. The content of interleukin$1 \beta$ (IL-1 $\beta$, JL18442), interleukin-6 (IL-6, JL20268), tumor necrosis factor- $\alpha$ (TNF- $\alpha$, JL10484), and monocyte chemotactic protein-1 (MCP-1, JL20304) in the kidney were also examined by ELISA kits (Shanghai Jianglai Industrial Limited by Share Ltd., China), respectively. All experimental procedures were carried out following the manufacturer's instructions.

2.5. Histological Assessment. The kidney tissues stored in $10 \%$ formalin solution were fixed with $4 \%$ paraformaldehyde and then dehydrated, embedded in paraffin wax, and sectioned into $4 \mu \mathrm{m}$ slices. The sections were stained with hematoxylineosin (H\&E), periodic acid-Schiff (PAS), and Masson's trichrome (Masson). The morphology of kidney tissues was observed using a light microscope for histopathological analysis. $\mathrm{H} \& \mathrm{E}$ staining results were reported descriptively. The glomerular injury was assessed by PAS and calculated from the integrated optical density (IOD), which is also equal to the positive area $\times$ average density. On the basis of Masson staining, tubulointerstitial fibrosis was assessed by the percentage of collagen area: collagen area $(\%)=$ the positive area/total area $\times 100$.

2.6. Detection of ROS. ROS production in the kidneys was further detected using DHE staining. Briefly, $10 \mu \mathrm{m}$ thick frozen renal tissues were obtained using a freezing microtome (CM1900, Leica, Germany) and samples were incubated with PBS for $15 \mathrm{~min}$. Subsequently, tissues were incubated with $5 \mathrm{mmol} / \mathrm{L}$ fluorescent-labeled DHE which was diluted by $1: 1000$ (KGAF019, KeyGEN BioTECH, 
Nanjing, China) in a lucifugal humidified chamber at $37^{\circ} \mathrm{C}$ for $30 \mathrm{~min}$, and then were stained with $4^{\prime}, 6^{\prime}$-diamidino-2pheny-lindole (DAPI, AS1075, Aspen Biological, Wuhan, China). Images were taken at 400x magnification under a fluorescence microscope (MicroPublisher, MP3.3-RTVCLR-10, Q-IMAGING, Canada). The quantitative analysis of the average DHE fluorescence intensity was measured by Image-Pro Plus 6.0 (IPP, Media Cybernetics, Rockville, MD, USA). Results were expressed by the ratio of the fluorescence intensity of DHE-positive area to the DAPI.

2.7. Immunofluorescence Imaging. Immunofluorescence staining of renal tissues was conducted to observe the extent of EMT. Frozen sections were placed in acetone ethanol and fixed for $20 \mathrm{~min}$ and then washed with PBS three times. Next, sections were placed in a 3\% solution of hydrogen peroxide and incubated in dark for $10 \mathrm{~min}$ at room temperature. These were then washed with PBS for three times and dried with $5 \%$ bovine serum albumin (BSA) for $20 \mathrm{~min}$. Samples were incubated with mouse anti- $\alpha$-SMA antibody (BM0002, 1:300, Boster) and mouse anti-E-cadherin antibody (GB12082, 1:150, Serviceio) at $4^{\circ} \mathrm{C}$ overnight, and then, goat anti-mouse antibodies were added for $1 \mathrm{~h}$. Subsequently, DAPI was applied for $5 \mathrm{~min}$. Stained sections were imaged at 400x magnification under a fluorescence microscope (MicroPublisher, MP3.3-RTV-CLR-10, Q-IMAGING, Canada). The further quantitative analysis of $\alpha$-SMA and Ecadherin was performed by IPP.

2.8. Cell Culture and Treatment. Human proximal tubular epithelial cell lines (HK-2 cells) were purchased from Nanjing Kaiji Biotechnology Development Co., Ltd. (Nanjing, Jiangsu, China) and cultured at $37^{\circ} \mathrm{C}$ in $5 \%$ humidified $\mathrm{CO}_{2}$ in Dulbecco's modified Eagle medium (DMEM)/Ham's F12 media ( $1: 1$, Gibco, Grand Island, NY, USA) containing $10 \%$ fetal bovine serum, $100 \mathrm{U} / \mathrm{mL}$ penicillin, and $100 \mu \mathrm{g} / \mathrm{mL}$ streptomycin (Beyotime, Nanjing, Jiangsu, China). The medium was changed every five days. HK-2 cells were cultured in 6-well plates and divided into four experimental groups: Group A: HK-2 cells were treated with $5.5 \mathrm{mM}$ Dglucose; Group B: HK-2 cells were treated with concentrations of $30 \mathrm{mM}$ D-glucose; Group C: HK-2 cell was treated with $30 \mathrm{mM}$ D-glucose plus $0.4 \mathrm{mg} / \mathrm{mL}$ YSPDF; and Group D: HK-2 cells were treated with $30 \mathrm{mM}$ D-glucose plus $0.4 \mathrm{mg} / \mathrm{mL}$ YSPDF and $10 \mu \mathrm{mol} \mathrm{Nrf2}$ inhibitor (ML385). After a $48 \mathrm{~h}$ incubation, the cells were lysed in M-PER mammalian protein extraction reagent (Thermo Fisher) for western blotting analysis.

2.9. Western Blot. Western blots were performed as previously described [25]. Total protein was extracted from kidney tissue in RIPA lysis buffer $(25 \mathrm{mM}$ Tris- $\mathrm{HCl}, 25 \mathrm{mM}$ $\mathrm{NaCl}, 0.5 \mathrm{mM}$ EDTA, $1 \%$ Triton X-100, and $0.1 \%$ SDS) with 1\% PMSF protease inhibitors (P1005, Beyotime Biotechnology, China) and phosphatase inhibitors (P1081, Beyotime Biotechnology, China) added. Equal amounts of protein were separated by $10-15 \%$ SDS-PAGE and then transferred to PVDF membranes (IPVH00010, Millipore, Germany). After blocking with $5 \%$ nonfat milk for $3 \mathrm{~h}$, the membranes were incubated with the primary antibodies including TGF- $\beta 1, \alpha$-SMA, Nrf2, HO-1, NQO1, ASC, caspase-1 (ab215715, ab32575, ab137550, ab13248, ab80588, ab175449, ab1872, Abcam, UK), p-Smad2, Smad2, p-Smad3, Smad3, E-cadherin, NLRP3 ((\#18338, \#5339, \#9520, \#9523, \#3195, \#15101, Cell Signaling Technology, USA), and cleaved caspase-1 (AF4005, Affinity Biosciences, USA) at $4^{\circ} \mathrm{C}$ overnight. Then, the membranes were washed 3 times with TBST and incubated with the secondary antibodies for $1 \mathrm{~h}$ at room temperature. After washing with TBST for a further three times, the protein bands were visualized by enhanced chemiluminescence (32134, Thermo, USA) solution and imaged with Automated Imaging System (Gene Gnome5, Synoptics Ltd, UK). GAPDH or $\beta$-actin was assumed to be similarly abundant in all samples and was used as a loading control.

2.10. Statistical Analysis. Statistical analysis was performed using SPSS 22.0 software (IBM Corporation, Armonk, New York, USA). All experimental data were expressed as the mean \pm SEM. Differences were determined by one-way ANOVA test and Dunnett's test. $p$ values less than 0.05 were considered as statistically significant.

\section{Results}

3.1. Quality Analysis of YSPDF. The aqueous extract of the mixture containing four Chinese medicinal materials was measured by HPLC. Six compounds $1-6$, with the retention time at $7.96 \mathrm{~min}, 26.81 \mathrm{~min}, 27.56 \mathrm{~min}, 30.40 \mathrm{~min}$, $32.74 \mathrm{~min}$, and $33.51 \mathrm{~min}$, respectively, were identified as (1) calycosin glucoside, (2) aloe-emodin emodin, (3) rhein, (4) emodin, (5) chrysophanol, and (6) physcion according to results of the HPLC chromatogram of YSPDF (Figure 1). The ratio of the six identified compounds was approximately $1: 1.9: 3.2: 2: 7.5: 1.2$.

3.2. YSPDF Improved Blood and Urine Biochemical Parameters in $d b / d b$ Mice. As shown in Table 2, the levels of FBG and the kidney index of the untreated $d b / d b$ mice were significantly increased compared with the control group, while the $d b / d b$ mice receiving YSPDF treatment were significantly reduced. However, there were no significant changes in body weight. Results indicated that YSPDF was able to reduce the fasting blood glucose levels of $d b / d b$ mice and potentially might have a protective effect on kidney.

Moreover, higher levels of SCr and $24 \mathrm{~h}$ UTP and lower levels of urine creatinine and creatinine clearance in $d b / d b$ mice were observed compared with control mice. The levels of serum TC, TG, and LDL were also markedly elevated in the untreated DKD group, while HDL level was decreased, demonstrating an impairment in renal function. In contrast, treatment with YSPDF significantly reduced SCr, $24 \mathrm{~h} \mathrm{UTP,}$ TC and TG levels compared to the untreated DKD group. YSPDF treatment also increased the levels of urine creatinine, creatinine clearance, and HDL of DKD mice. These above results demonstrated that YSPDF may not only prevent kidney damage and improve renal function but also reduce levels of blood lipids. 


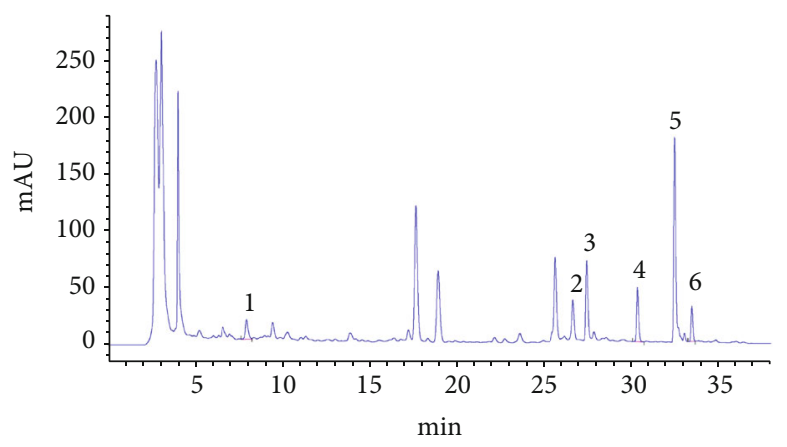

(a)

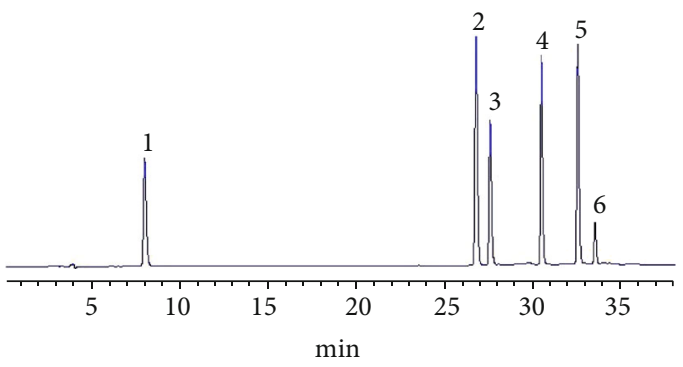

(b)

FIGURE 1: (a) HPLC chromatogram of YSPDF: (1) calycosin glucoside, (2) aloe-emodin emodin, (3) rhein, (4) emodin, (5) chrysophanol, and (6) physcion. (b) HPLC chromatogram of standard solution: (1) calycosin glucoside, (2) aloe-emodin emodin, (3) rhein, (4) emodin, (5) chrysophanol, and (6) physcion.

TABLE 2: YSPDF improved the levels of various biochemical parameters in $d b / d b$ mice.

\begin{tabular}{lccc}
\hline Biochemical parameters & NC & DKD & DKD+YSPDF \\
\hline Final body weight $(\mathrm{g})$ & $29.28 \pm 2.74$ & $46.42 \pm 5.31^{\# \# \#}$ & $43.76 \pm 3.21$ \\
Kidney index $(\%)$ & $0.96 \pm 0.11$ & $1.33 \pm 0.32^{\# \#}$ & $1.07 \pm 0.42^{*}$ \\
Fasting blood glucose $(\mathrm{mmol} / \mathrm{L})$ & $4.42 \pm 0.74$ & $23.78 \pm 5.61^{\# \# \#}$ & $15.26 \pm 4.64^{* *}$ \\
Serum creatinine $(\mu \mathrm{mol} / \mathrm{L})$ & $2.59 \pm 0.41$ & $5.52 \pm 0.51^{\# \# \#}$ & $18.29 \pm 4.11^{\# \#}$ \\
Urea nitrogen $(\mathrm{mmol} / \mathrm{L})$ & $6.01 \pm 0.14$ & $5.87 \pm 1.54^{\# \# \#}$ & $9.36 \pm 1.83^{* *}$ \\
24h urine protein $(\mathrm{mg} / \mathrm{L})$ & $1.37 \pm 0.36$ & $0.36 \pm 0.12^{\# \# \#}$ & $1.96 \pm 0.87^{* * *}$ \\
Urine creatinine $(\mathrm{mmol} / \mathrm{L})$ & $2.42 \pm 0.56$ & $0.93 \pm 0.15^{\# \#}$ & $0.81 \pm 0.31^{* * *}$ \\
Creatinine clearance $(\mathrm{mL} / \mathrm{min})$ & $1.82 \pm 0.62$ & $7.03 \pm 0.91^{\# \# \#}$ & $1.46 \pm 0.77^{* *}$ \\
Total cholesterol $(\mathrm{mmol} / \mathrm{L})$ & $3.86 \pm 0.44$ & $3.68 \pm 0.79^{\# \# \#}$ & $4.66 \pm 1.09^{* * *}$ \\
Triglyceride $(\mathrm{mmol} / \mathrm{L})$ & $1.14 \pm 0.41$ & $1.07 \pm 0.38^{\#}$ & $2.43 \pm 0.49^{*}$ \\
HDL $(\mathrm{mmol} / \mathrm{L})$ & $1.77 \pm 0.35$ & $0.51 \pm 0.15^{\#}$ & $2.25 \pm 0.75^{*}$ \\
LDL $(\mathrm{mmol} / \mathrm{L})$ & $0.30 \pm 0.11$ & $0.59 \pm 0.47$ \\
\hline
\end{tabular}

$\mathrm{NC}$ refers to the normal control group; DKD refers to diabetic kidney disease group. Data were expressed as the mean \pm SEM $(n=8) .{ }^{\#} p<0.05,{ }^{\# \#} p<0.01$, and ${ }_{\# \# \#} p<0.001$ compared with the normal control group; ${ }^{*} p<0.05,{ }^{* *} p<0.01$, and ${ }^{* * *} p<0.001$ compared with the DKD group.

3.3. YSPDF Alleviated the Histopathological Changes of Kidney in $d b / d b$ Mice. The histopathological alterations were investigated in renal tissues via H\&E staining, PAS staining, and Masson staining. Generally, $\mathrm{H} \& \mathrm{E}$ staining was used to observe the lesions in kidney tissues, PAS staining was used to examine the glomerular sclerosis index, and Masson staining was used to assess the renal fibrosis. As shown with H\&E- and PAS-stained kidney tissues (Figure 2(a), 2(b)), compared with the control group, the untreated DKD group exhibited kidney damage, including abnormal glomerular structure, interstitial edema and hyperemia, thickening of the glomerular basement membrane, and an increase in the amount of inflammatory cell infiltration and fibrosis. In contrast, glomerular injury, renal damage and inflammatory responses were significantly reduced by YSPDF treatment.

Masson staining (Figures 2(a) and 2(b)) showed that the basement membrane of renal tissue in DKD mice was much bluer, and the distribution of Masson-positive areas was significantly enlarged, indicating the increasing content of collagen fibers and collagen deposition, signs of fibrosis. These phenomena were significantly improved after treatment with YSPDF. Results indicated that YSPDF could ameliorate tubulointerstitial damage, renal fibrosis in DKD mice compared with untreated animals.

3.4. YSPDF Mitigated Renal Fibrosis in Kidney of db/db Mice by Inhibiting EMT. To estimate whether YSPDF could reduce $\mathrm{EMT}$ in the kidneys of $\mathrm{db} / \mathrm{db}$ mice, we determined the levels of EMT-associated proteins. Immunofluorescence imaging shows the protein expression (Figure 3(a)) and fluorescence intensity (Figure 3(c)) of $\alpha$-SMA was considerably increased in the kidneys of untreated DKD mice, and this was attenuated with YSPDF treatment. Further results showed that YSPDF administration markedly inhibited Ecadherin expression as compared to untreated DKD mice (Figure 3(b)). The fluorescence intensity of E-cadherin (Figure 3(c)) was reduced in untreated DKD mice, with YSPDF treatment decreasing the amount of this reduction. These results indicated that YSPDF administration might inhibit renal fibrosis by inhibiting EMT. 

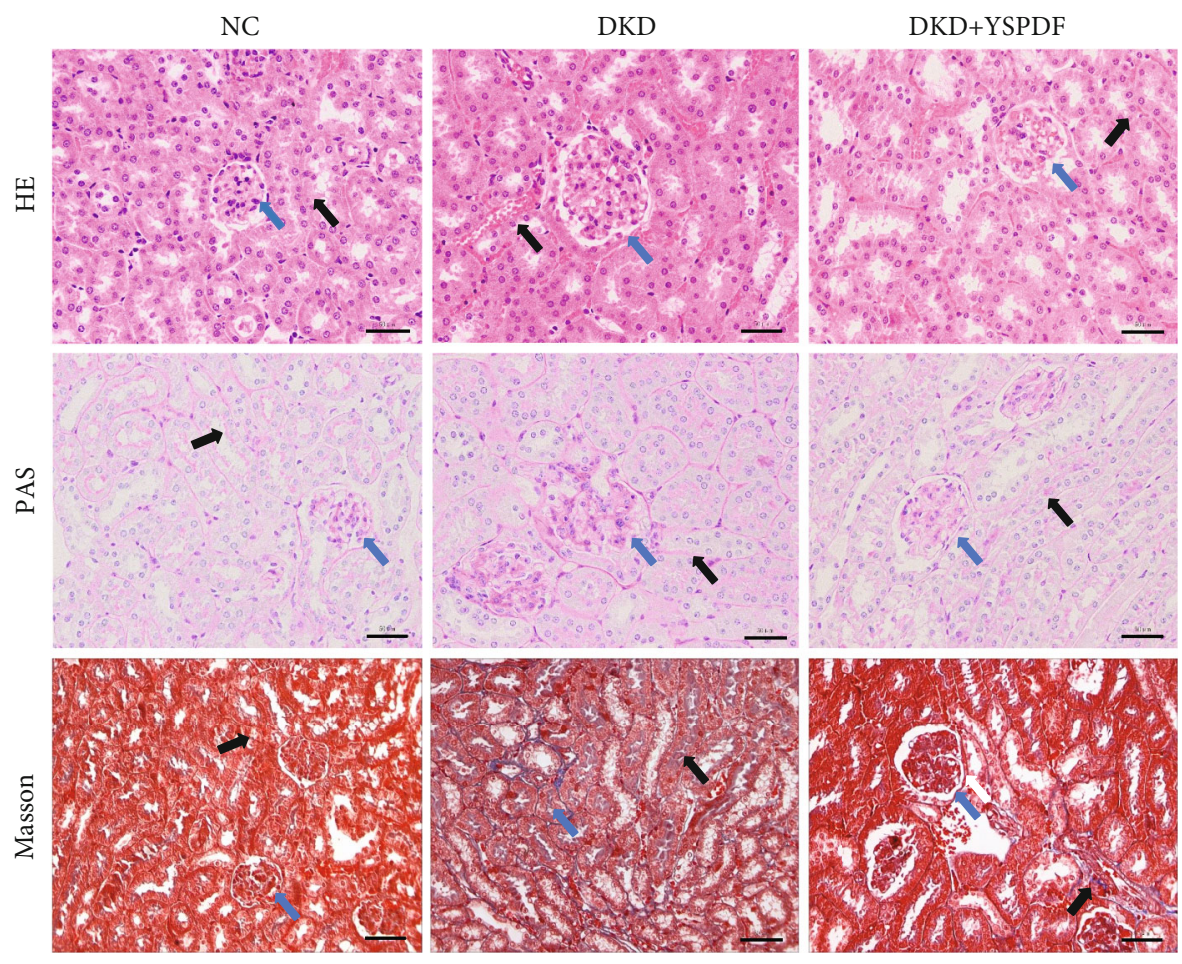

(a)
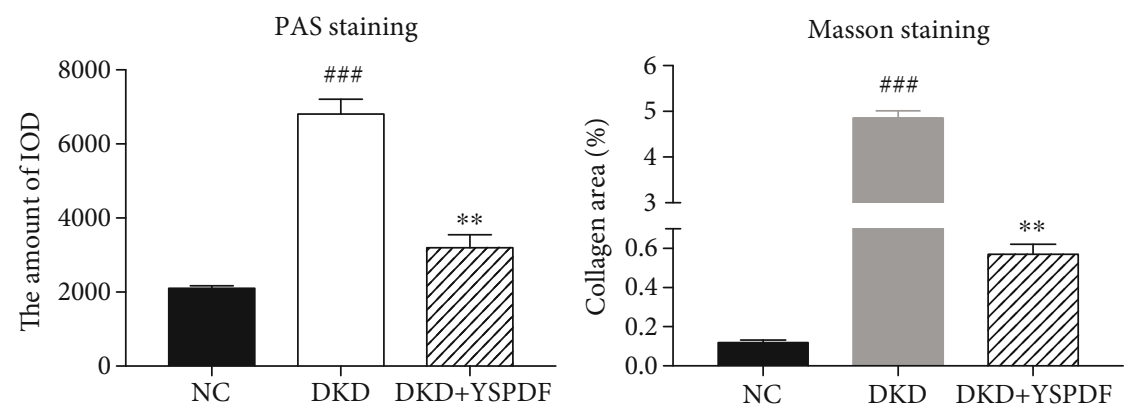

(b)

FIgURE 2: Effect of YSPDF on histopathological changes of the kidney in $d b / d b$ mice. (a) Representative images of H\&E, PAS, and Masson staining of kidney tissues were shown (400x). Each bar indicates $50 \mu \mathrm{m}$. (b) Glomerular injury using PAS staining and tubulointerstitial fibrosis using Masson staining. Blue arrows show the glomeruli, and the black arrows show kidney tubules. Data were presented as the mean \pm SEM; $n=3 ;{ }^{\# \# \#} p<0.001$ compared with the normal control group; ${ }^{* *} p<0.01$ compared with the DKD group.

3.5. YSPDF Inhibited Renal Oxidative Stress in db/db Mice. Oxidative stress has been shown to play a crucial role in diabetes-induced kidney injury [8]. Therefore, indicators of oxidative stress injury including ROS level, MDA level, GSH level, and SOD and CAT activities in kidney tissues were measured. As shown in Figure 4(a), levels of ROS and MDA in untreated $d b / d b$ mice were significantly elevated compared with the control group $(p<0.01)$, with this elevation being inhibited by YSPDF treatment. In contrast, the levels of GSH, SOD, and CAT were markedly decreased $(p<0.01)$. Treatment with YSPDF attenuated this decrease for all three parameters. DHE staining showed that YSPDF significantly inhibited ROS production in the kidneys of $d b / d b$ mice as compared with the untreated DKD group mice (Figure 4(b)).
3.6. YSPDF Alleviated the Production of Inflammatory Cytokines in the Kidneys of db/db Mice. To confirm the effect of YSPDF on inflammation and renal damage, the expressions of proinflammatory cytokines including TNF- $\alpha$, IL- 6 , IL- $1 \beta$, and MCP- 1 were measured. ELISA assays (Figure 5) showed that the levels of TNF- $\alpha$, IL- 6 , IL- $1 \beta$, and MCP-1 in the kidney tissues of DKD mice were significantly higher than those of control mice. YSPDF treatment significantly decreased the levels of these inflammatory factors.

3.7. YSPDF Treatment Inhibited Renal Fibrosis in the Kidneys of $d b / d b$ Mice by Blocking EMT via Suppressing the TGF- $\beta 1 /$ Smad2/3 Signaling Pathway. TGF- $\beta 1$ has been shown to play a crucial role in the development of renal cell hypertrophy and tubulointerstitial fibrosis via its downstream 

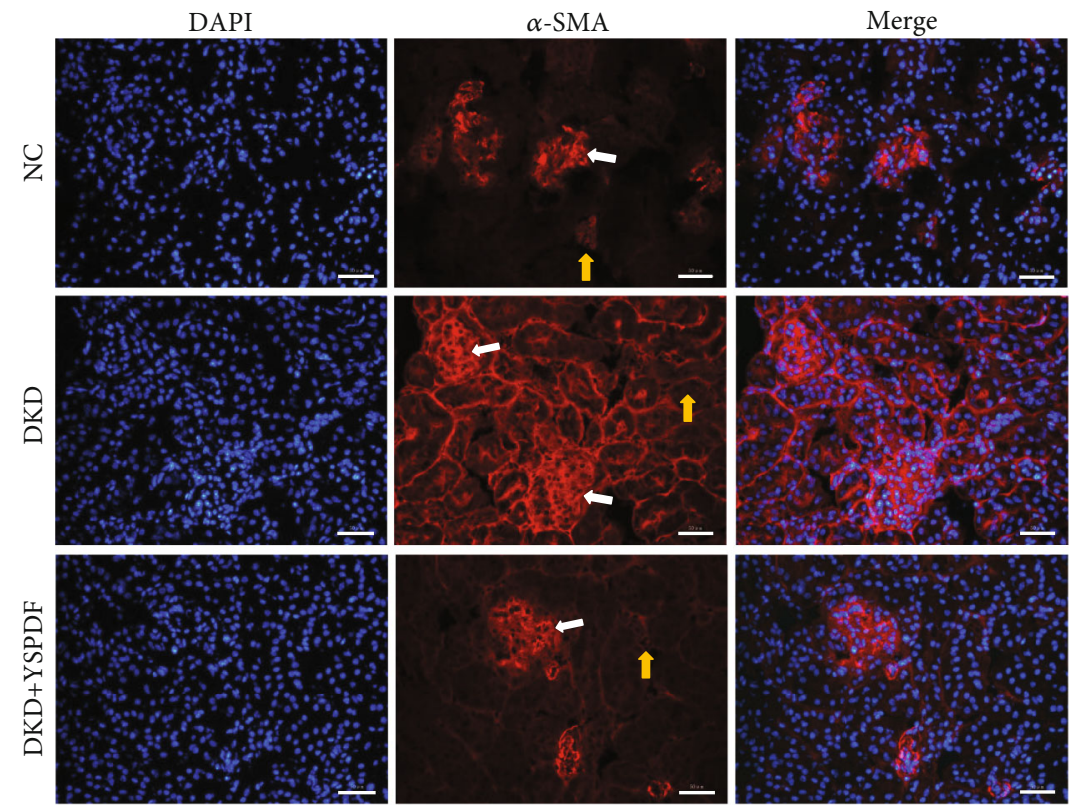

(a)
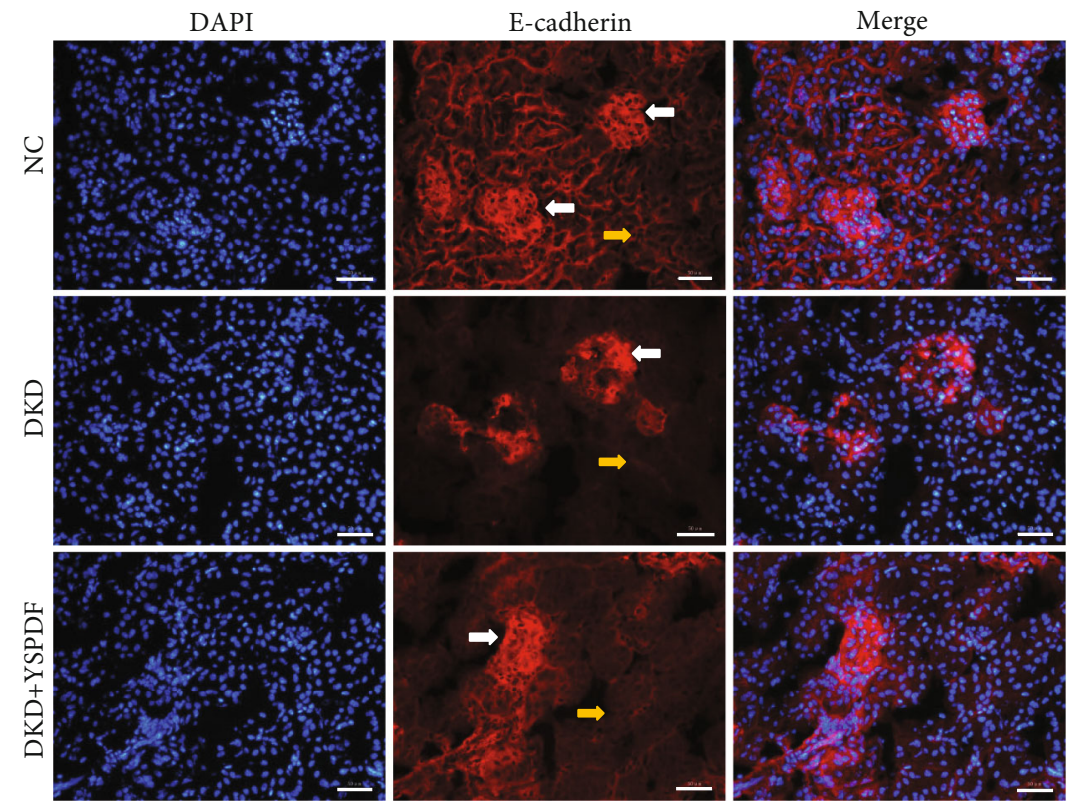

(b)
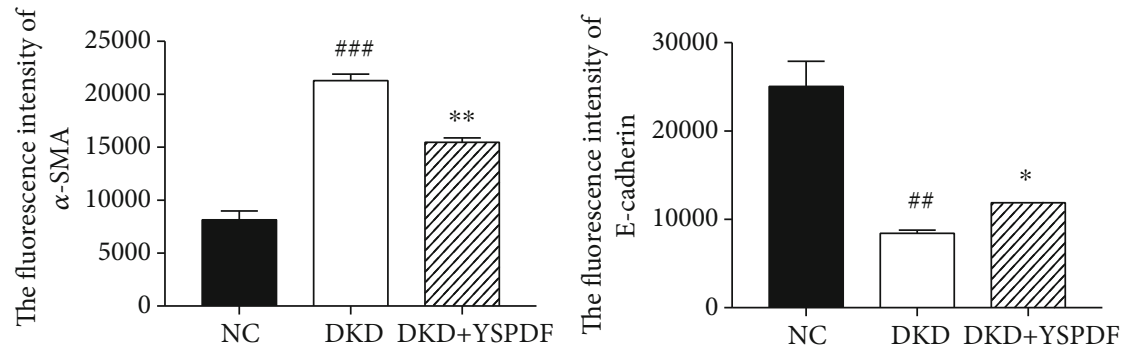

(c)

Figure 3: Representative immunofluorescence images of (a) $\alpha$-SMA (red) and (b) E-cadherin (red). Each bar indicates $50 \mu$ m. (c) The fluorescence intensity was measured by IPP, and results were expressed by the ratio of fluorescence intensity of the positive area to DAPI. White arrows show the glomeruli, and the yellow arrows show kidney tubules. Data were presented as the mean \pm SEM; $n=3$; ${ }^{\# \#} p<0.01$ and ${ }^{\# \# \#} p<0.001$ compared with the normal control group; ${ }^{*} p<0.05$ and ${ }^{* *} p<0.01$ compared with the DKD group. 

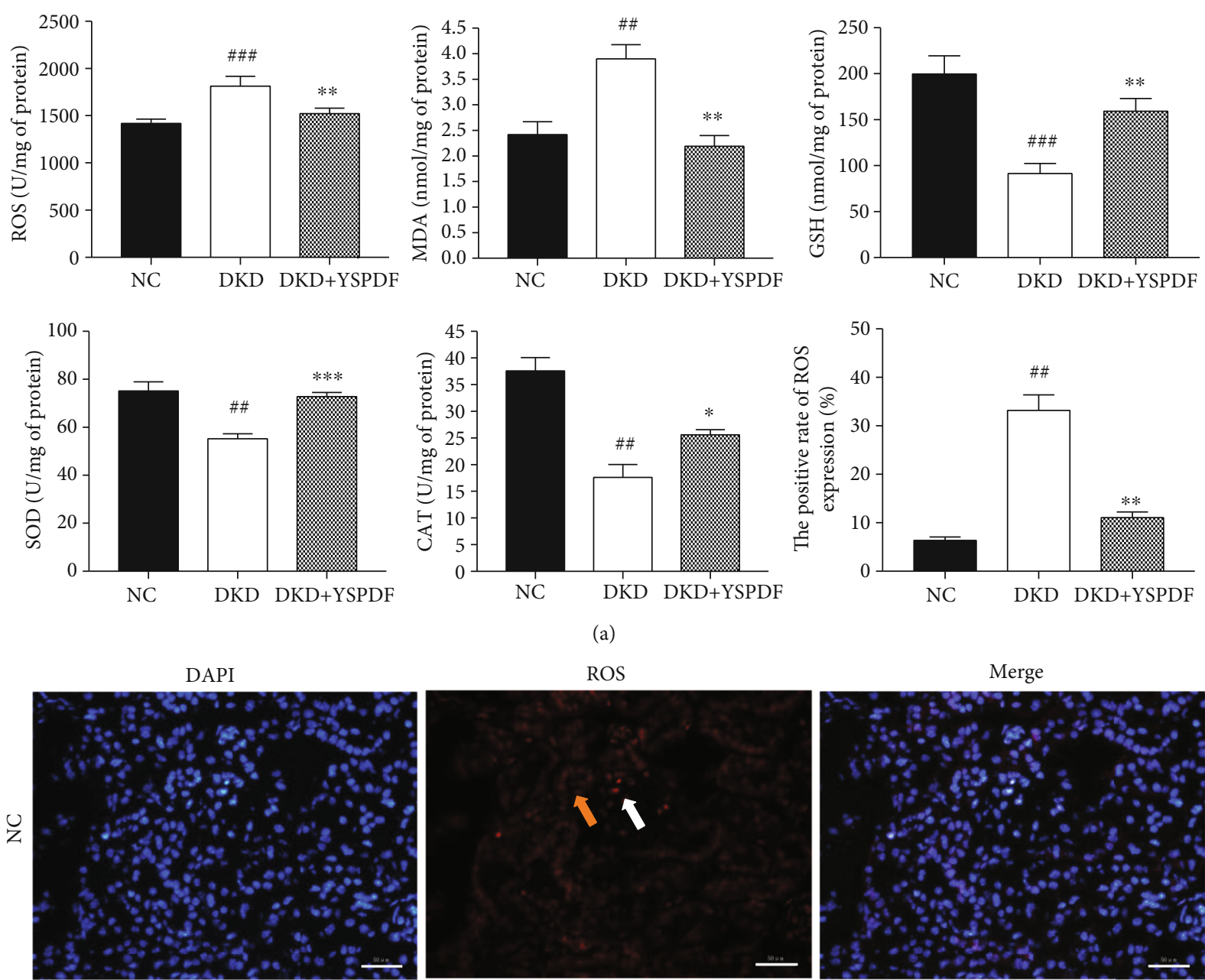

(a)
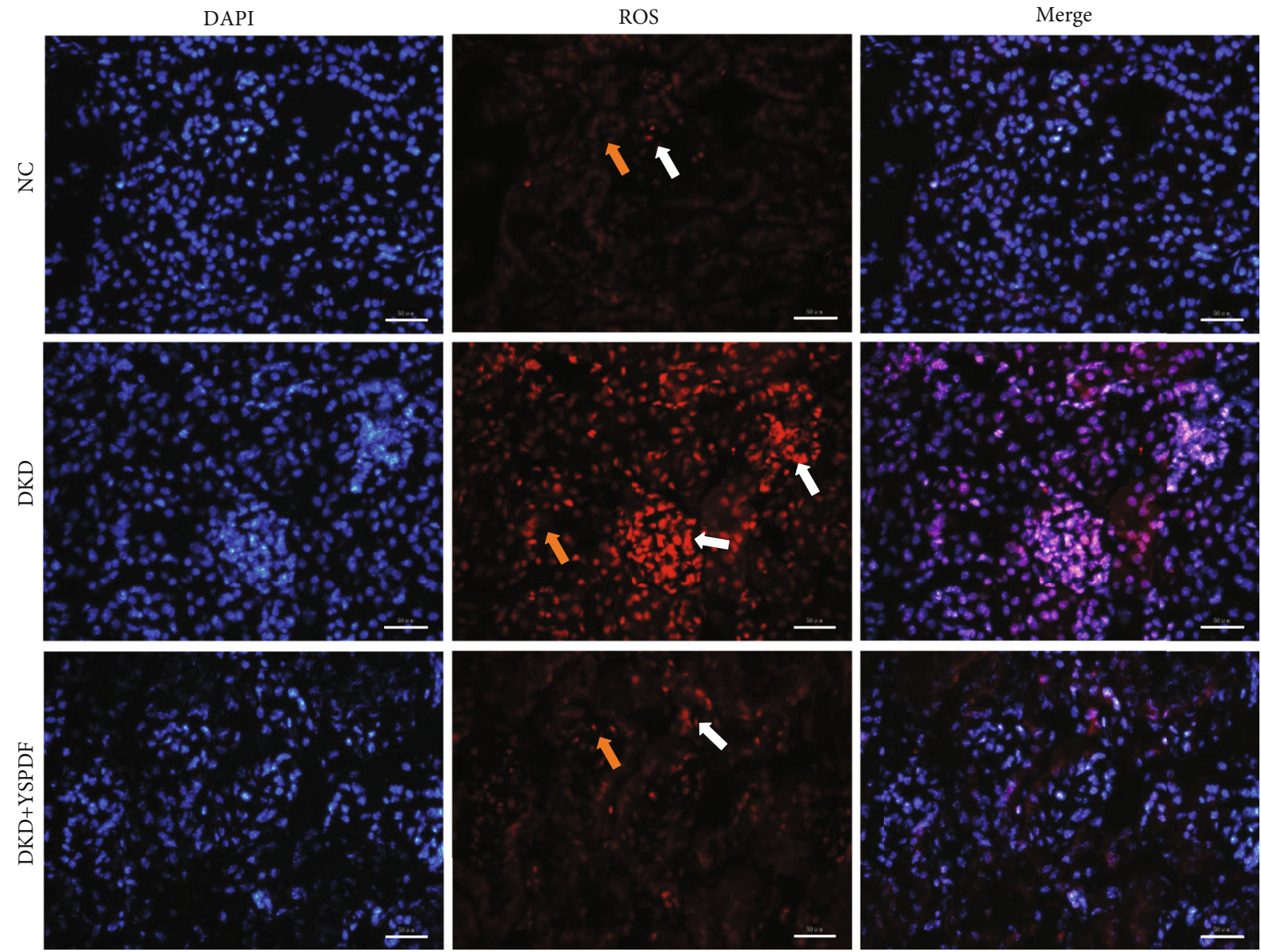

(b)

FIGURE 4: YSPDF ameliorated oxidative stress in $d b / d b$ mice. (a) Oxidative stress parameters including ROS, MDA, GSH, SOD, and CAT were determined by commercial assay kits. DHE fluorescence intensity was measured by IPP, and results were expressed by the ratio of fluorescence intensity of the DHE-positive area to the DAPI. (b) ROS level was determined by DHE staining (400x). Each bar indicates $50 \mu \mathrm{m}$. White arrows show the glomeruli, and the yellow arrows show kidney tubules. ${ }^{\#} p<0.01$ and ${ }^{\# \#} p<0.001$ compared with the normal control group; ${ }^{*} p<0.05,{ }^{* *} p<0.01$, and ${ }^{* * *} p<0.001$ compared with the DKD group. 

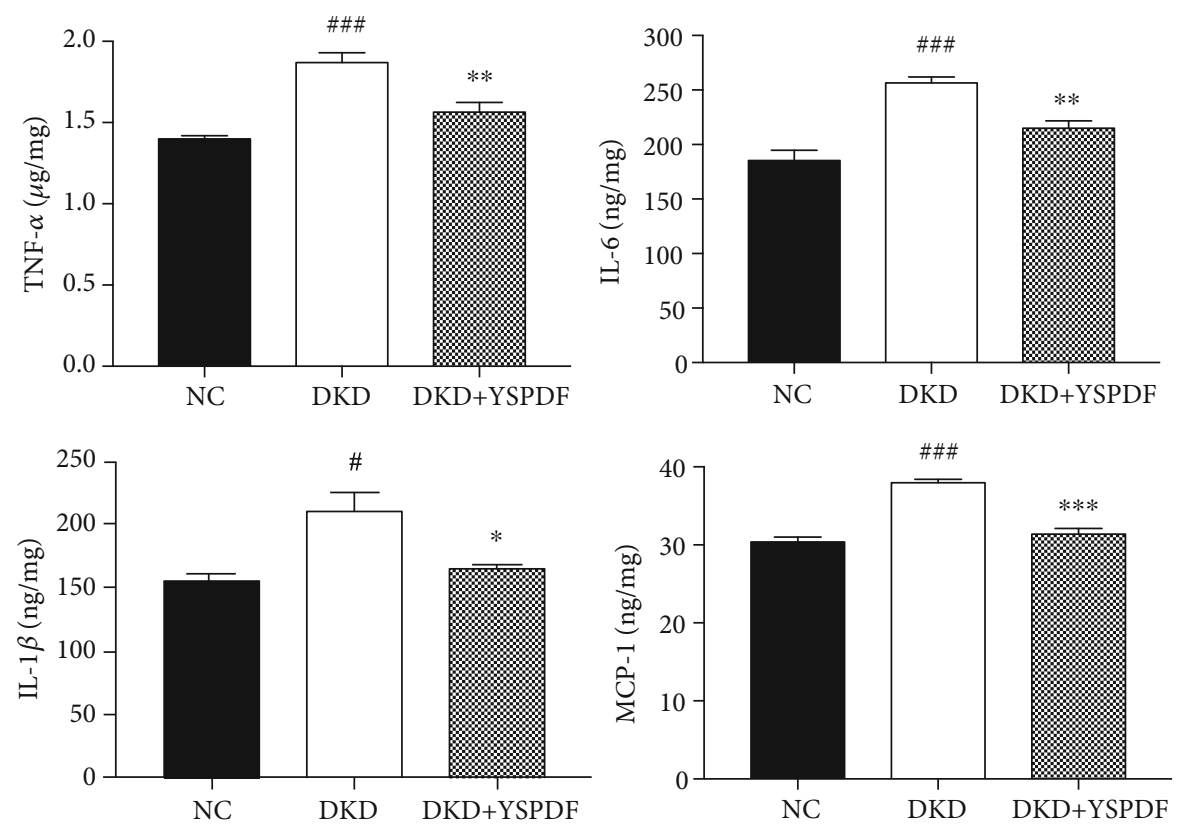

FIGURE 5: YSPDF inhibited the production of inflammatory factors in $d b / d b$ mice. Proinflammatory factors including TNF- $\alpha$, IL-6, IL- $1 \beta$, and MCP-1 were detected by ELISA assay. ${ }^{\#} p<0.05$ and ${ }^{\# \#} p<0.001$ compared with the normal control group; ${ }^{*} p<0.05,{ }^{* *} p<0.01$, and *** $p<0.001$ compared with the DKD group.

Smad-dependent pathway and the regulation of the expression of EMT proteins such as $\alpha$-SMA and E-cadherin [20]. As indicated in Figure 6(a), the expression levels of TGF- $\beta 1$, $\mathrm{p}$-Smad2, p-Smad3, and $\alpha$-SMA were markedly increased in the kidney tissues of untreated DKD mice as compared to the control group. However, the expression levels of these proteins were significantly suppressed by YSPDF. Additionally, the level of E-cadherin expression was downregulated in the untreated DKD group, which was partially recovered by YSPDF treatment. Therefore, our results further indicated that YSPDF may suppress the progression of renal fibrosis via inhibiting EMT by blocking the TGF- $\beta 1$ signaling pathway.

3.8. YSPDF Protected Kidneys from Injury by Activating Nrf2 and Inhibiting NLRP3 Inflammasome Signaling Pathways in $d b / d b$ Mice. We investigated further the underlying molecular mechanism explaining the protection of YSPDF on DKD by analyzing its effects on the important antioxidant signaling Nrf2 pathway. The key proteins of the Nrf2 pathway, including Nrf2, HO-1 and NQO1, were examined by western blot. The expressions of Nrf2 and its downstream factors HO-1 and NQO1 were distinctly reduced in untreated DKD mice (Figure 6(b)). Treatment with YSPDF partially reversed these changes. We also evaluated the effect of YSPDF on the inhibition of the renal NLRP3 inflammasome, which has been reported to be important in the development of renal injury [12]. Western blot results showed that the expression levels of inflammasome proteins including NLRP3, ASC, caspase- 1 , and cleaved caspase- 1 in the kidneys of DKD mice were significantly elevated compared with the control group (Figure 6(c)). In contrast, YSPDF partially rescued the levels of NLRP3, ASC, caspase-1, and cleaved caspase-1 when compared to the untreated DKD group. Collectively, these results demonstrated that YSPDF might ameliorate oxidative stress and inflammation by activating the Nrf2 pathway and by inhibiting the NLRP3 inflammasome pathway.

3.9. YSPDF Inhibited EMT and NLRP3 Inflammasome Signaling Pathway by Activating Nrf2 Pathway in High Glucose- (HG-) Induced HK-2 Cells. To support the previous in vivo results, HK-2 cells in HG conditions were treated with YSPDF (Figure 7). Results demonstrated that HG significantly increased the expressions of $\alpha$-SMA, TGF- $\beta 1$, and NLRP 3 and decreased the expression of Nrf2 in HK-2 cells as compared to the NC group $(p<0.01)$. Nevertheless, compared with the HG group, $\alpha$-SMA, TGF- $\beta 1$, and NLRP3 expressions in the YSPDF group were decreased, whereas that of Nrf2 was increased. Next, to determine whether Nrf2 was required for YSPDF protection from HG-induced harmful effects, a Nrf2 inhibitor (ML 385) was used to inhibit Nrf2 expression in HK-2 cells. As shown in Figure 7, ML385 reduced Nrf2 expression in YSPDFtreated cells, which resulted in increasing expressions of $\alpha$ SMA, TGF- $\beta 1$, and NLRP3. This result suggested that YSPDF-induced activation of the Nrf2 pathway was prevented by the Nrf2 inhibitor. Accordingly, YSPDF induced inhibition of the NLRP3 inflammasome pathway, TGF- $\beta 1$ pathway, and EMT were partly reversed in HG-exposed HK-2 cells treated with ML385. These findings suggested that YSPDF protected HK-2 cells from HG-induced injury in a manner dependent on Nrf2 activation.

\section{Discussion}

The pathogenesis of DKD is complicated and accumulating studies show that oxidative stress, inflammation, and EMT 

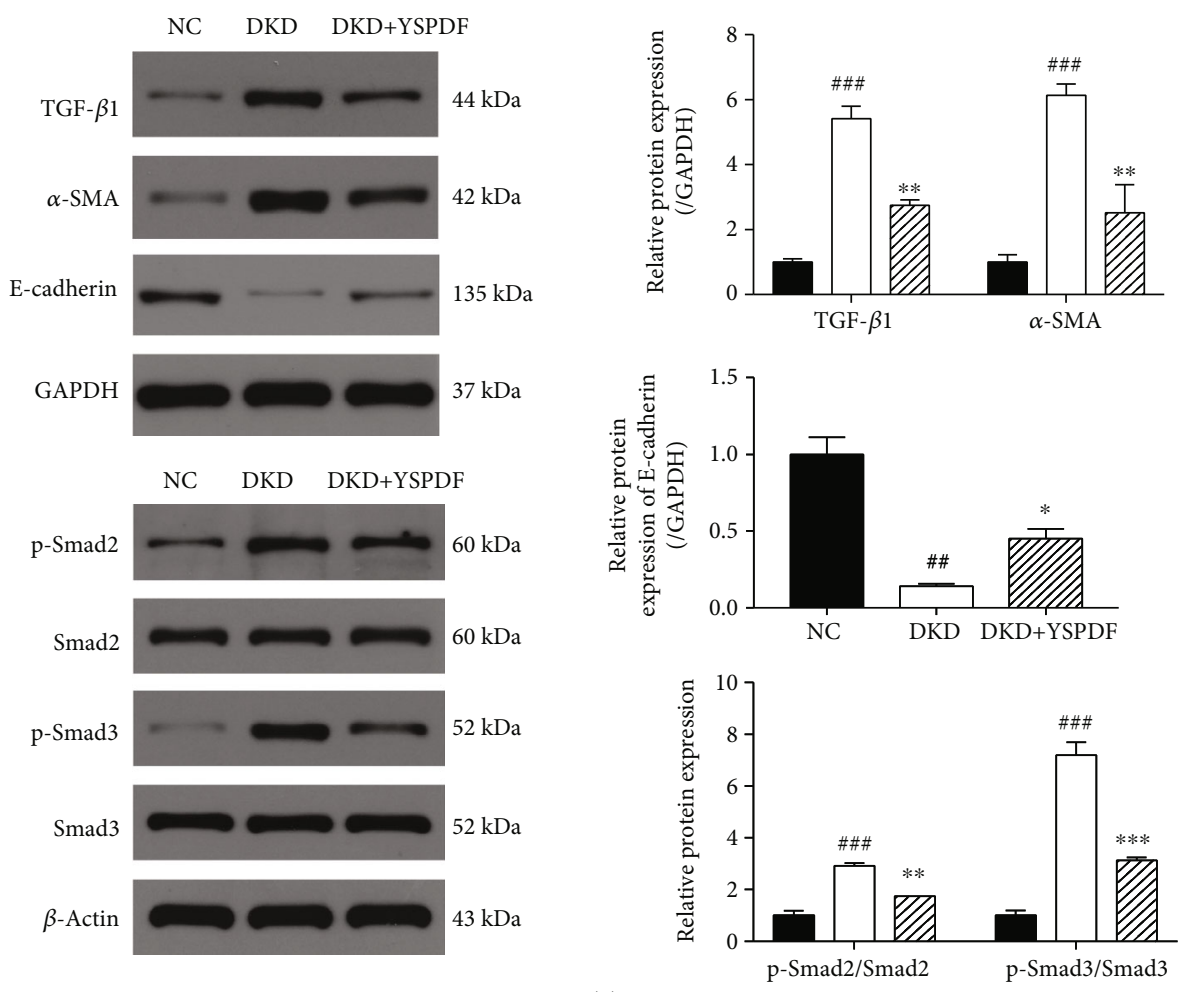

(a)
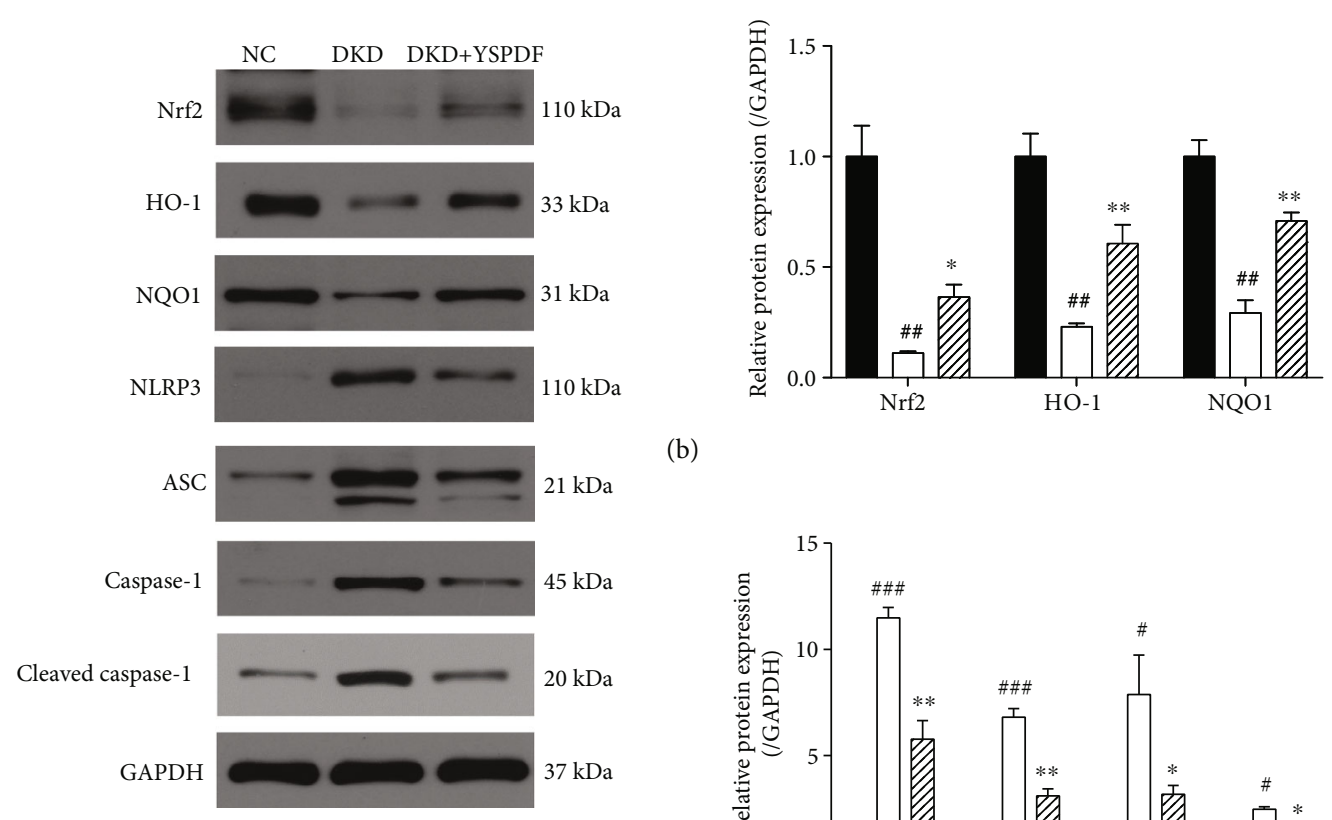

(b)

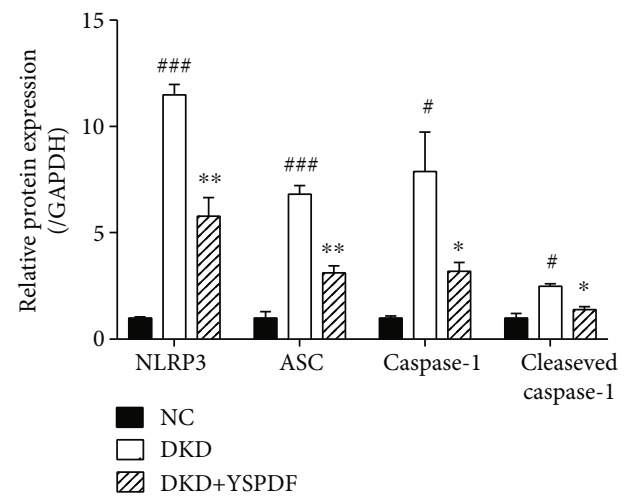

(c)

FIGURE 6: YSPDF treatment regulated the expressions of TGF- $\beta 1$, p-Smad2, Smad2, p-Smad3, Smad3, $\alpha$-SMA, E-cadherin, Nrf2, HO-1, NQO1, NLRP3, ASC, caspase-1, and cleaved caspase-1 in the kidneys of $d b / d b$ mice. Protein expression levels were normalized to the levels of either GAPDH or $\beta$-actin. The data were analyzed using a one-way ANOVA and expressed as the mean \pm SEM $(n=3)$. ${ }^{\#} p<0.05,{ }^{\# \#} p<0.01$, and ${ }^{\# \#} p<0.001$ compared with the normal control group; ${ }^{*} p<0.05$ and ${ }^{* *} p<0.01$ compared with the DKD group. 

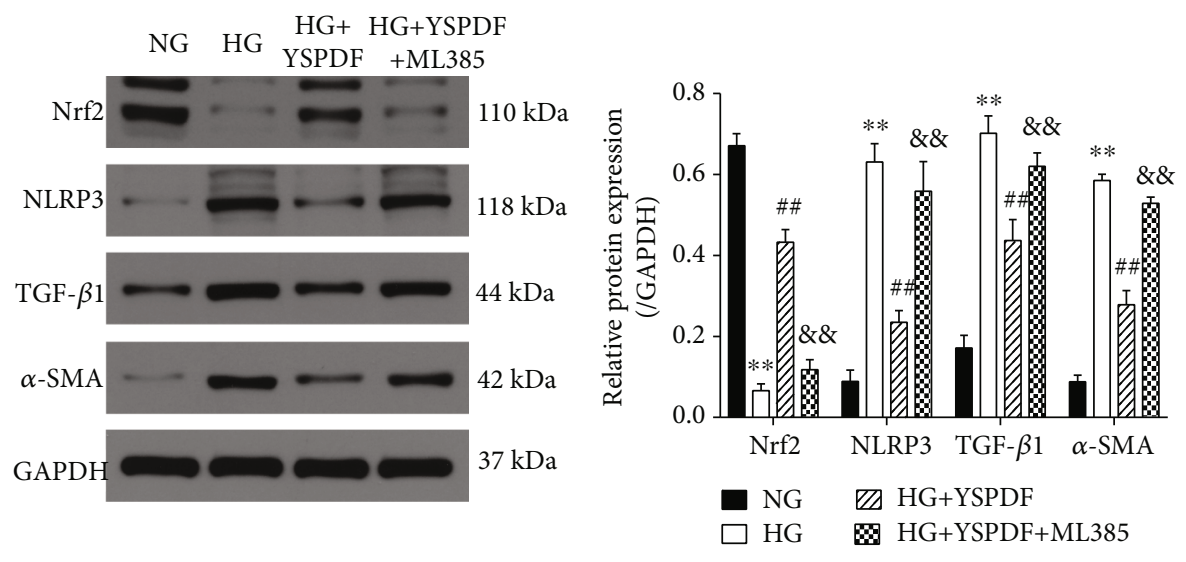

Figure 7: The expressions of Nrf2, NLRP3, TGF- $\beta 1$, and $\alpha$-SMA in HK-2 cells treated by NG, HG, HG+YSPDF, and HG+YSPDF + ML385. Protein expression levels were normalized to the levels of GAPDH. The data were analyzed using a one-way ANOVA and expressed as the mean $\pm \operatorname{SEM}(n=3) .{ }^{* *} p<0.01$ compared with the NG group; ${ }^{\# \#} p<0.01$ compared with HG group; ${ }^{\& \&} p<0.01$ compared with the HG+YSPDF group.

played important roles in disease progression, in addition to a disorder in blood glucose and lipid metabolism [26-28]. Consequently, revealing more information on the mechanisms behind kidney injury in DKD and finding new drugs or therapeutic targets has become a research hotspot globally.

In recent years, traditional Chinese medicine (TCM) has shown broad and promising application prospects in the treatment of DKD due to its advantage of having low toxicology and high efficiency [3, 29]. YSPDF has been used in clinical practice in our hospital for twenty years with no reports of significant side effects [24]. Results showed that YSPDF, coated aldehyde oxystarch capsules and a combination therapy of YSPDF with coated aldehyde oxystarch capsules could reduce SCr and uric acid, increase eGFR, and protect renal function in patients with CKD3 and CKD4, with the cotreated group being more significant than in the YSPDF and coated aldehyde oxystarch capsule-treated groups [30]. The acute toxicity assay results of YSPDF in healthy Sprague Dawley (SD) rats showed that none of the rats died and the visceral organs had no changes in gross anatomy. Histopathological analysis of the liver and kidney showed no toxicity in the experimental animals, and no significant changes were found in biochemical parameters (including white blood cell count, red white blood cell count, hemoglobin, platelet count, alamine aminotransferase, aspartate transaminase, serum creatinine, and urea nitrogen) when compared to those in the control group [24]. All results suggest that YSPDF is safe and effective and will not cause renal function damage.

In the present study, we used an experimental animal model of DKD using $d b / d b$ mice. A significant increase of the kidney index, FBG, SCr, $24 \mathrm{~h}$ UTP, serum cholesterols, and triglycerides were observed in DKD mice, which is consistent with previous studies [14, 31]. Several clinical studies have shown that long-term hyperglycemia was the most critical element that mediated the progressive tissue damage and functional decline characterizing diabetic complications in DKD [14, 31]. Long-term hyperglycemia induces insulin resistance and oxidative stress. Insulin resistance activates signaling pathways, such as P38 and extracellular signalregulated kinase (ERK) pathways, to increase the expression of TGF- $\beta$, while increased oxidative stress cooperates with the activated polyol pathway to stimulate the protein kinase $\mathrm{C}$ (PKC) pathway $[14,31]$. All of these contribute to the deposition of ECM and leads to glomerulosclerosis and tubular interstitial fibrosis. Thus, the controlling of blood glucose levels could effectively delay the progression of DKD [14, 31]. Here, we find that YSPDF was able to significantly decrease blood glucose levels in $d b / d b$ mice, which indicates that the protective effect of YSPDF might be related its hypoglycemic activity. Moreover, histopathological results showed renal fibrosis as well as accumulation of inflammatory cell infiltration in the untreated DKD mice. On the contrary, YSPDF treatment not only partially restored blood and urine parameters but also helped ameliorate the pathological changes in the kidneys of the diabetic mice. These results demonstrated that YSPDF was an effective treatment for protecting against and alleviating renal damage and renal fibrosis.

TGF- $\beta 1 /$ Smad signaling is currently recognized as one of the most potent profibrogenic factors and has been shown to be a crucial mediator in EMT $[32,33]$. Increased expression of $\alpha$-SMA and a decreased expression of E-cadherin were major hallmarks of EMT. TGF- $\beta 1$ is able to activate Smad2/3 via phosphorylation, accelerating the process of fibrosis [34]. In this study, the expression levels of TGF- $\beta 1$, $\mathrm{p}$-Smad2/Smad2, p-Smad3/Smad3, and $\alpha$-SMA were significantly upregulated in untreated $d b / d b$ kidney tissues, with a significant dampening of this affect seen in the YSPDFtreated group. Moreover, YSPDF significantly increased the expression of E-cadherin compared to the untreated $d b / d b$ mice from the results of western blot and immunofluorescence assay. Our results were consistent with a previous observation that TGF- $\beta 1$ might induce EMT and ROS generation, where it was shown that treatment with an 


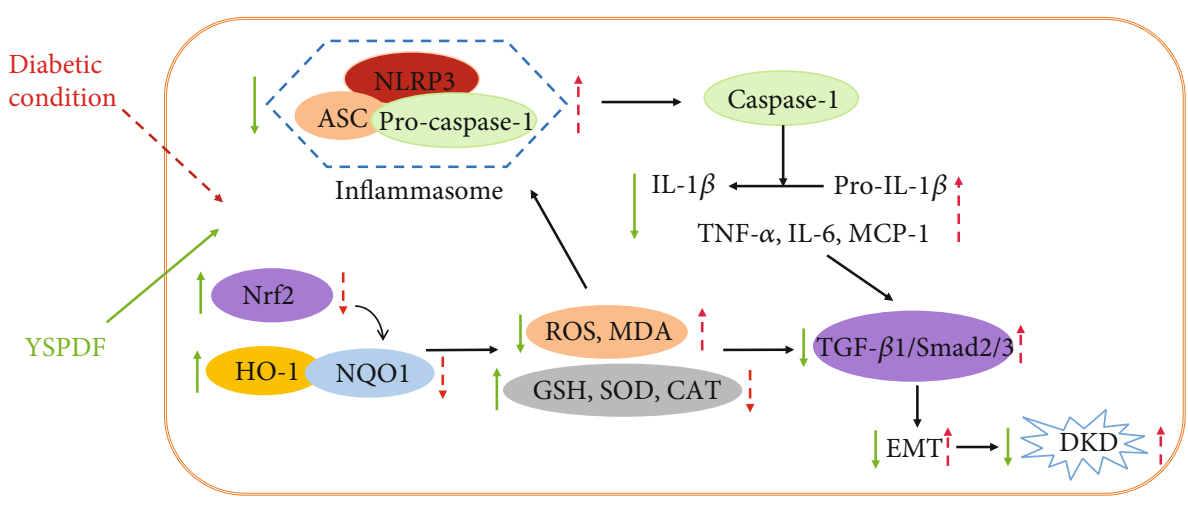

FIgUre 8: The possible molecular mechanisms of YSPDF on DKD.

antioxidant was able to markedly prevent EMT in HK-2 cells [32]. These experimental results suggest that inhibition of tubular EMT to mitigate renal fibrosis via inhibiting the TGF- $\beta 1 /$ Smad signaling pathway might be one of the mechanisms by which YSPDF was able to alleviate kidney damage.

The excessive production of ROS in renal cells under the condition of hyperglycemia is considered an important pathological mechanism for DKD [31]. In the present study, our results found that the levels of GSH, SOD, and CAT activities were downregulated in the kidneys of untreated DKD mice, whereas ROS and MDA increased, demonstrating severe oxidative damage occurring in the kidneys of $d b / d b$ mice. YSPDF treatment partially reversed these changes and distinctly suppressed ROS production, implying that the renal protective effect of YSPDF might be associated with its strong antioxidative properties.

Nrf2 is a critical redox-sensitive transcription factor, and once activated, it transfers to the nucleus and activates the transcription of many antioxidant genes, such as HO-1 and NQO1, thus reducing oxidative stress [35-37]. It was reported that $\mathrm{Nrf} / \mathrm{HO}-1$ was able to inhibit the pathological process of $\mathrm{DKD}$ by decreasing oxidative stress and inflammation [38]. Our findings were consistent with those of other studies showing that the expression levels of Nrf2, and its downstream targets HO-1 and NQO1 were significantly decreased in the untreated DKD group compared with the nondiabetic control group. This effect was significantly attenuated with YSPDF administration, indicating that the Nrf2 pathway may be involved in the antioxidative and protective effect of YSPDF against DKD. Previous research has reported that treatment of a rat glomerular mesangial cell line (HBZY-1) with an Nrf2 inhibitor (ML385) suppressed the activation of Nrf2, HO-1, and NQO1 and that this abolished the protective effect of treatments reducing oxidative stress [39]. Similarly, we showed that YSPDF activated Nrf2 in HG-induced HK-2 cells, which resulted in the reductions of $\alpha$-SMA and TGF- $\beta 1$ expression. Nevertheless, YSPDF-mediated activation of the Nrf2 pathway was further inhibited by ML385. These findings support the hypothesis that YSPDF ameliorates renal damage in DKD mice and HG-exposed HK-2 cells by activating the Nrf2 pathway.
There is evidence that hyperglycemia increases the release of proinflammatory cytokines and promotes the synthesis of ECM proteins in glomeruli cells, leading to renal inflammation and fibrosis [39]. Several proinflammatory cytokines, such as TNF- $\alpha$, IL-6, IL- $1 \beta$, and IL-18 were reported to participate in the pathogenesis of DKD [39]. In this study, $d b / d b$ mice had increased levels of TNF- $\alpha$, IL-6, IL-1 $\beta$, and MCP-1 in kidney tissues, consistent with published studies $[38,40]$. YSPDF treatment helped reverse this increase, suggesting its inhibitory effects on inflammation.

Accumulating studies have shown that the NLRP3 inflammasome, which is composed of the NLRP3 protein, caspase-1, and ASC, plays a pivotal role in DKD kidney inflammation, especially in podocytes, endothelial cells, and renal tubular epithelial cells $[41,42]$. Once cells were subjected to stress, NLRP3 quickly activated and recruited ASC and pro-caspase-1, leading to activation of caspase-1, thereby resulting in the secretion of pro-inflammation cytokines such as IL- $1 \beta$ and IL-18 in DKD [28, 43]. Moreover, oxidative stress was also reported to be the key element of activating the NLRP3 inflammasome [44, 45]. ROSinduced NLRP3 activation was shown to accelerate the kidney damage in mice fed a high-fat diet [45].

According to our western blot and ELISA assay results, expression levels of NLRP3, ASC, and caspase-1 proteins and IL- $1 \beta$ in the kidneys of DKD mice were markedly increased. Furthermore, an abnormal glomerular structure and a large amount of inflammatory cell infiltration and fibrosis were also observed in the DKD group. However, these phenomena were significantly normalized in mice treated with YSPDF. In line with our findings, NLRP3 deficiency has been shown to mitigate renal inflammation and fibrosis in DKD [15]. Inhibition of NLRP3 inflammasome activation by silencing NLRP3/ASC or repressing caspase-1 activity has been shown to decrease the production of IL$1 \beta$ and alleviate kidney damage [16]. Several lines of evidence have revealed that diabetes-induced renal NLRP3 inflammasome activation, TGF- $\beta 1 / \mathrm{Smad} 3$ activation, and ECM protein accumulation were inhibited in NLRP3 knockout mice [15]. In addition, Nrf2 activation could also inhibit the NLRP3 pathway. In this study, treatment with YSPDF significantly increased Nrf2 expression and reduced NLRP3 expression in HG-exposed HK-2 cells, whereas this effect 
of YSPDF was abolished by a Nrf2 inhibitor. Therefore, we suggest that YSPDF may protect kidneys from damage in DKD via suppression of the production of inflammatory cytokines and by inhibiting the activation of the NLRP3 inflammasome via activating the Nrf2 pathway.

\section{Conclusion}

In conclusion, as a traditional Chinese medicine preparation, YSPDF exhibited a renal protective effect in $d b / d b$ mice. Moreover, we demonstrated that YSPDF can attenuate oxidative stress and inflammatory states in the kidneys of DKD mice via activating the Nrf2 pathway, inhibiting the NLRP3 inflammasome pathway, and reducing renal tubular EMT via inhibiting the TGF- $\beta 1 /$ Smad pathway, thus reducing kidney injury. Cell experiments showed that YSPDF inhibited EMT and the NLRP3 inflammasome in HGexposed HK-2 cells, possibly through activation of Nrf2. The possible mechanisms of YSPDF treatment of DKD were summarized in Figure 8. Therefore, we conclude that YSPDF may be a potential and promising drug to treat DKD.

\section{Abbreviations}

YSPDF: $\quad$ Yi Shen Pai Du formula

DKD: $\quad$ Diabetic kidney disease

CKD: $\quad$ Chronic kidney disease

EMT: Epithelial-to-mesenchymal transition

DN: Diabetic nephropathy

ESRD: End-stage renal failure

ECM: $\quad$ Extracellular matrix

TGF- $\beta 1$ : Transforming growth factor- $\beta 1$

$\alpha$-SMA: $\quad \alpha$-Smooth muscle actin

Nrf2: $\quad$ Nuclear factor-erythroid 2-related factor 2

NLRP3: Nucleotide binding and oligomerization domain-like receptor family pyrin domaincontaining 3

ASC: $\quad$ Apoptosis-associated speck-like protein containing a caspase recruitment domain

ROS: $\quad$ Reactive oxygen species

RP-HPLC: Reversed phase high-performance liquid chromatography

UCr: Urinary creatinine

FBG: $\quad$ Fasting blood glucose

SCr: $\quad$ Serum creatinine

BUN: Blood urea nitrogen

TC: $\quad$ Total cholesterol

TG: $\quad$ Total triglycerides

HDL: High-density lipoprotein

LDL: $\quad$ Low-density lipoprotein

GSH: $\quad$ Glutathione

MDA: $\quad$ Malondialdehyde

SOD: $\quad$ Superoxide dismutase

ELISA: Enzyme-linked immunosorbent assay

IL-1 $\beta$ : Interleukin-1 $\beta$

IL-6: $\quad$ Interleukin-6

TNF- $\alpha$ : Tumor necrosis factor- $\alpha$

MCP-1: Monocyte chemotactic protein-1

HE: Hematoxylineosin
PAS: $\quad$ Periodic acid-Schiff

DAPI: $\quad 4^{\prime}, 6^{\prime}$-Diamidino-2-pheny-lindole.

\section{Data Availability}

The data used to support the findings of this study are available from the corresponding author upon request.

\section{Conflicts of Interest}

There are no conflicts of interest to declare.

\section{Authors' Contributions}

QZ, CS, and BD contributed to the experimental design. XL contributed to animal experiments and biochemical analysis. MAS contributed to manuscript writing, critical review of results, and revising. QZ and $\mathrm{BD}$ contributed to molecular biology experiments, data analysis, result interpretation, and writing. All authors have read, revised, and approved the final manuscript. Qilin Zhang and Xiaocui Liu both are equal first author.

\section{Acknowledgments}

This work was supported by the National Natural Science Foundation of China (Grant No. 81803799) and Hubei Province Natural Science Foundation of China (Grant No. 2017CFB273). MAS is supported by an Advance Queensland Industry Research Fellowship, Mater Foundation, Equity Trustees, and the L G McCallam Est and George Weaber Trusts.

\section{References}

[1] N. H. Cho, J. E. Shaw, S. Karuranga et al., "IDF Diabetes Atlas: global estimates of diabetes prevalence for 2017 and projections for 2045," Diabetes Research and Clinical Practice, vol. 138, pp. 271-281, 2018.

[2] H. Yaribeygi, N. Katsiki, A. E. Butler, and A. Sahebkar, "Effects of antidiabetic drugs on NLRP3 inflammasome activity, with a focus on diabetic kidneys," Drug Discovery Today, vol. 24, no. 1, pp. 256-262, 2019.

[3] Z. Lu, Y. Zhong, W. Liu, L. Xiang, and Y. Deng, "The efficacy and mechanism of Chinese herbal medicine on diabetic kidney disease," Journal Diabetes Research, vol. 2019, article 2697672, pp. 1-14, 2019.

[4] J. Wei, Y. Shi, Y. Hou et al., "Knockdown of thioredoxininteracting protein ameliorates high glucose-induced epithelial to mesenchymal transition in renal tubular epithelial cells," Cellular Signalling, vol. 25, no. 12, pp. 2788-2796, 2013.

[5] I. Loeffler and G. Wolf, "Epithelial-to-mesenchymal transition in diabetic nephropathy: fact or fiction?," Cell, vol. 4, no. 4, pp. 631-652, 2015.

[6] H. Y. Lan, "Transforming growth factor- $\beta /$ Smad signalling in diabetic nephropathy," Clinical and Experimental Pharmacology \& Physiology, vol. 39, no. 8, pp. 731-738, 2012.

[7] Z. Wang, Z. Han, J. Tao et al., "Role of endothelial-tomesenchymal transition induced by TGF- $\beta 1$ in transplant kidney interstitial fibrosis," Journal of Cellular and Molecular Medicine, vol. 21, no. 10, pp. 2359-2369, 2017. 
[8] S. M. Fernandes, P. M. Cordeiro, M. Watanabe, C. D. Fonseca, and M. F. Vattimo, "The role of oxidative stress in streptozotocin-induced diabetic nephropathy in rats," Archives of Endocrinology and Metabolism, vol. 60, no. 5, pp. 443-449, 2016.

[9] J. Donate-Correa, D. Luis-Rodriguez, E. Martin-Nunez et al., "Inflammatory targets in diabetic nephropathy," Journal of Clinical Medicine, vol. 9, no. 2, pp. 1-22, 2020.

[10] E. H. Kobayashi, T. Suzuki, R. Funayama et al., "Nrf2 suppresses macrophage inflammatory response by blocking proinflammatory cytokine transcription," Nature Communications, vol. 7, no. 1, article 11624, 2016.

[11] I. Lazaro, L. Lopez-Sanz, S. Bernal et al., "Nrf2 activation provides atheroprotection in diabetic mice through concerted upregulation of antioxidant, anti-inflammatory, and autophagy mechanisms," Frontiers in Pharmacology, vol. 9, p. 819, 2018.

[12] Y. Y. Qiu and L. Q. Tang, "Roles of the NLRP3 inflammasome in the pathogenesis of diabetic nephropathy," Pharmacological Research, vol. 114, pp. 251-264, 2016.

[13] L. Du, L. Wang, B. Wang et al., "A novel compound AB38b attenuates oxidative stress and ECM protein accumulation in kidneys of diabetic mice through modulation of Keap1/Nrf2 signaling," Acta Pharmacologica Sinica, vol. 41, no. 3, pp. 358-372, 2020.

[14] Y. Zhu, C. Zhu, H. Yang, J. Deng, and D. Fan, "Protective effect of ginsenoside $\operatorname{Rg} 5$ against kidney injury via inhibition of NLRP3 inflammasome activation and the MAPK signaling pathway in high-fat diet/streptozotocin-induced diabetic mice," Pharmacological Research, vol. 155, p. 104746, 2020.

[15] M. Wu, W. Han, S. Song et al., "NLRP3 deficiency ameliorates renal inflammation and fibrosis in diabetic mice," Molecular and Cellular Endocrinology, vol. 478, pp. 115-125, 2018.

[16] Y. Liu, Z. Xu, F. Ma, Y. Jia, and G. Wang, "Knockdown of TLR4 attenuates high glucose-induced podocyte injury via the NALP3/ASC/Caspase-1 signaling pathway," Biomedicine \& Pharmacotherapy, vol. 107, pp. 1393-1401, 2018.

[17] C. Y. Zhang, J. Y. Zhu, Y. Ye et al., "Erhuang Formula ameliorates renal damage in adenine-induced chronic renal failure rats via inhibiting inflammatory and fibrotic responses," Biomedicine \& Pharmacotherapy, vol. 95, pp. 520-528, 2017.

[18] L. Yu, Y. Yang, Q. He, and D. Chen, "Preparation of nephritis dripping pills and its pharmacodynamic studies," Herald of Medicine, vol. 34, no. 5, pp. 617-620, 2015.

[19] J. Lv, Z. Wang, Y. Wang et al., "Renoprotective effect of the Shen-Yan-Fang-Shuai formula by inhibiting TNF- $\alpha / N F-\kappa B$ signaling pathway in diabetic rats," Journal Diabetes Research, vol. 2017, article 4319057, pp. 1-11, 2017.

[20] L. Ma, H. Li, S. Zhang et al., "Emodin ameliorates renal fibrosis in rats via TGF- $\beta 1 /$ Smad signaling pathway and function study of Smurf 2," International Urology and Nephrology, vol. 50, no. 2, pp. 373-382, 2018.

[21] A. Hosseini, H. Mollazadeh, M. S. Amiri, H. R. Sadeghnia, and A. Ghorbani, "Effects of a standardized extract of_Rheum turkestanicum Janischew root on diabetic changes in the kidney, liver and heart of streptozotocin-induced diabetic rats," Biomedicine \& Pharmacotherapy, vol. 86, pp. 605-611, 2017.

[22] Y. Xie, F. Lan, J. Zhao, and W. Shi, "Hirudin improves renal interstitial fibrosis by reducing renal tubule injury and inflammation in unilateral ureteral obstruction (UUO) mice," International Immunopharmacology, vol. 81, p. 106249, 2020.
[23] B. Deng, Q. Wang, C. Zhou, and Q. Zhang, "Study on determination of active components content and fingerprint of Yishen Paidu pills," Drug Evaluation Research, vol. 42, no. 4, pp. 658662, 2019.

[24] Q. Zhang, B. Deng, C. Zhou, and Q. Wang, "TLC identification and acute toxicity assay of Yishen Paidu pills," China Pharmacist, vol. 22, no. 8, pp. 1523-1526, 2019.

[25] Y. Qiu, X. Jiang, D. Liu et al., “The hypoglycemic and renal protection properties of crocin via oxidative stress-regulated NF- $\kappa \mathrm{B}$ signaling in $\mathrm{db} / \mathrm{db}$ mice," Frontiers in Pharmacology, vol. 11, p. 541, 2020.

[26] S. T. Chou and S. T. Tseng, "Oxidative stress markers in type 2 diabetes patients with diabetic nephropathy," Clinical and Experimental Nephrology, vol. 21, no. 2, pp. 283-292, 2017.

[27] H. Yaribeygi, S. L. Atkin, L. E. Simental-Mendía, G. E. Barreto, and A. Sahebkar, "Anti-inflammatory effects of resolvins in diabetic nephropathy: mechanistic pathways," Journal of Cellular Physiology, vol. 234, no. 9, pp. 14873-14882, 2019.

[28] W. Han, Q. Ma, Y. Liu et al., "Huangkui capsule alleviates renal tubular epithelial-mesenchymal transition in diabetic nephropathy via inhibiting NLRP3 inflammasome activation and TLR4/NF- $\kappa \mathrm{B}$ signaling," Phytomedicine, vol. 57, pp. 203-214, 2019.

[29] L. Zhang, L. Yang, J. Shergis et al., "Chinese herbal medicine for diabetic kidney disease: a systematic review and metaanalysis of randomised placebo-controlled trials," BMJ Open, vol. 9, no. 4, article e025653, 2019.

[30] Q. Wen, Retrospective study on stage 3 and stage 4 of chronic renal diseasae treated by renal failure pill, Huazhong University of Science and Technology, 2017.

[31] L. Du, J. Wang, Y. Chen et al., "Novel biphenyl diester derivative AB-38b inhibits NLRP3 inflammasome through Nrf2 activation in diabetic nephropathy," Cell Biology and Toxicology, vol. 36, no. 3, pp. 243-260, 2020.

[32] S. Song, D. Qiu, F. Luo et al., "Knockdown of NLRP3 alleviates high glucose or TGFB1-induced EMT in human renal tubular cells," Journal of Molecular Endocrinology, vol. 61, no. 3, pp. 101-113, 2018.

[33] J. Jin, Z. Zhang, J. Chen, Y. Liu, Q. Chen, and Q. Wang, "Jixuepaidu Tang-1 inhibits epithelial-mesenchymal transition and alleviates renal damage in DN mice through suppressing long non-coding RNA LOC498759," Cell Cycle, vol. 18, no. 22, pp. 3125-3136, 2019.

[34] S. Das, S. Dewanjee, T. K. Dua et al., "Carnosic acid attenuates cadmium induced nephrotoxicity by inhibiting oxidative stress, promoting Nrf2/HO-1 signalling and impairing TGF$\beta 1 /$ Smad/collagen IV signalling," Molecules, vol. 24 , no. 22 , p. 4176, 2019.

[35] J. Li, H. Jiang, P. Wu et al., "Toxicological effects of deltamethrin on quail cerebrum: weakened antioxidant defense and enhanced apoptosis," Environmental Pollution, vol. 286, article 117319, 2021.

[36] S. Li, X. Zheng, X. Zhang et al., "Exploring the liver fibrosis induced by deltamethrin exposure in quails and elucidating the protective mechanism of resveratrol," Ecotoxicology and Environmental Safety, vol. 207, p. 111501, 2021.

[37] N. Deng, H. Jiang, P. Wu et al., "Inhibition of the Nrf2/p38MAPK pathway involved in deltamethrin-induced apoptosis and fibrosis in quail kidney," Food and Chemical Toxicology, vol. 155, article 112382, 2021. 
[38] C. Lu, G. Fan, and D. Wang, “Akebia Saponin D ameliorated kidney injury and exerted anti-inflammatory and antiapoptotic effects in diabetic nephropathy by activation of NRF2/HO-1 and inhibition of NF-KB pathway," International Immunopharmacology, vol. 84, p. 106467, 2020.

[39] X. Wu, C. Pan, R. Chen, S. Zhang, Y. Zhai, and H. Guo, "BML111 attenuates high glucose-induced inflammation, oxidative stress and reduces extracellular matrix accumulation via targeting Nrf2 in rat glomerular mesangial cells," International Immunopharmacology, vol. 79, p. 106108, 2020.

[40] S. M. Yang, S. M. Ka, H. L. Wu et al., "Thrombomodulin domain 1 ameliorates diabetic nephropathy in mice via antiNF- $\kappa$ B/NLRP3 inflammasome-mediated inflammation, enhancement of NRF2 antioxidant activity and inhibition of apoptosis," Diabetologia, vol. 57, no. 2, pp. 424-434, 2014.

[41] M. Lu, N. Yin, W. Liu, X. Cui, S. Chen, and E. Wang, "Curcumin ameliorates diabetic nephropathy by suppressing NLRP3 inflammasome signaling," BioMed Research International, vol. 2017, Article ID 1516985, 10 pages, 2017.

[42] R. Hu, M. Q. Wang, S. H. Ni et al., "Salidroside ameliorates endothelial inflammation and oxidative stress by regulating the AMPK/NF- $\kappa \mathrm{B} / \mathrm{NLRP} 3$ signaling pathway in AGEsinduced HUVECs," European Journal of Pharmacology, vol. 867, p. 172797, 2020.

[43] E. K. Jo, J. K. Kim, D. M. Shin, and C. Sasakawa, "Molecular mechanisms regulating NLRP3 inflammasome activation," Cellular \& Molecular Immunology, vol. 13, no. 2, pp. 148159, 2016.

[44] Q. Shan, Y. Zheng, J. Lu et al., "Purple sweet potato color ameliorates kidney damage via inhibiting oxidative stress mediated NLRP3 inflammasome activation in high fat diet mice," Food and Chemical Toxicology, vol. 69, pp. 339-346, 2014.

[45] G. H. Zheng, Q. Shan, J. J. Mu et al., "Purple sweet potato color attenuates kidney damage by blocking VEGFR2/ROS/NLRP3 signaling in high-fat diet-treated mice," Oxidative Medicine and Cellular Longevity, vol. 2019, Article ID 5189819, 16 pages, 2019. 